\title{
Tunable THz radiation source from dielectric loaded waveguide excited by nonrelativistic electron bunch trains
}

\author{
Weiwei Li, Zhigang He, ${ }^{*}$ Yalin Lu, ${ }^{\dagger}$ Ruixuan Huang, Weihao Liu, Qika Jia, and Lin Wang \\ National Synchrotron Radiation Laboratory, University of Science and Technology of China, \\ Hefei, Anhui 230029, China
}

(Received 13 January 2016; published 6 October 2016)

\begin{abstract}
We propose a novel scheme to generate a tunable narrow-band $\mathrm{THz}$ radiation. In this scheme, a train of laser pulses with $\mathrm{THz}$ repetition rate is used to drive a photocathode direct current (DC) gun, leading to the emission of a train of electron bunches. The electron bunch train is subsequently accelerated by the gun field and applied to selectively excite one of the modes in the dielectric loaded waveguide (DLW) structure, which is located downstream the DC gun. Thanks to the tunability of the repetition rate of laser pulses and the gun voltage, a tunable narrow-band $\mathrm{THz}$ radiation source can be obtained. This proposed source has the advantages of compactness, robustness and relatively high power.
\end{abstract}

DOI: 10.1103/PhysRevAccelBeams.19.104701

\section{INTRODUCTION}

Terahertz $(\mathrm{THz})$ radiation, which lies in the frequency gap between the infrared and microwaves, typically referred to as the frequencies from $100 \mathrm{GHz}$ to $30 \mathrm{THz}$, is finding use in an increasingly wide variety of applications $[1,2]$. However lack of the desirable radiation source has always been the main bottleneck for the development of the terahertz applications [3].

Accelerator-based $\mathrm{THz}$ sources can provide high-power $\mathrm{THz}$ radiation due to the generation of ultrashort relativistic electron beams. These sources can be typically categorized into two types: broad-band and narrow-band. Broad-band $\mathrm{THz}$ sources are often based on coherent synchrotron radiation (CSR) [4-6] and coherent transition radiation (CTR) [7-10] excited by ultrashort electron bunches. Narrow-band $\mathrm{THz}$ radiation can be obtained by using FEL technology [11-15], dielectric loaded waveguide (DLW) $[16,17]$ and other slow wave structures $[18,19]$ as emitter, and train of electron bunches as driving beam [20-26]. These kinds of sources can generate the radiation of above megawatt peak power with a spectrum covering the whole terahertz band, yet their practical applications are limited by the large equipment, enormous cost and complicated peripherals, such as microwave power source, radiation shielding, etc. Besides, these sources cannot achieve a high average power since the accelerators are usually operated at a repetition rate lower than $100 \mathrm{~Hz}$

\footnotetext{
corresponding author.

hezhg@ustc.edu.cn

corresponding author. yllu@ustc.edu.cn
}

Published by the American Physical Society under the terms of the Creative Commons Attribution 3.0 License. Further distribution of this work must maintain attribution to the author $(s)$ and the published article's title, journal citation, and DOI. except for the superconducting based ones. $\mathrm{THz}$ radiation sources can also be realized by methods based on nonrelativistic electron beams, such as backward-wave oscillator (BWO) and Smith-Purcell radiation (SPR) driven by $\mathrm{THz}$ electron bunch trains [27], which overcome the large volume of the accelerators. However, the traditional BWO can only generate radiation at a frequency much lower than $1 \mathrm{THz}$ due to the limitations of very tiny-size structure and extremely high starting current density [28], and the transfer efficiency of SPR is low. Thus, an electron-beamdriven $\mathrm{THz}$ source that is compact, easily achievable, efficient and tunable, would be in great demand.

The DLW supports a discrete set of modes (due to the waveguide boundaries) with phase velocity equal to the electron beam velocity that are thus capable of efficient energy exchange with the beam. It has been used for generating intense $\mathrm{THz}$ radiation [16,17], studying dielectric wakefield accelerator [29-31], forming density modulation in electron beam [32], and measuring the electron bunch length [33]. In this paper, we propose to selectively excite one of the lowest monopole modes in a DLW structure by nonrelativistic electron bunch trains as a narrow-band $\mathrm{THz}$ source. Thanks to the tunability of the beam velocity and the spacing between microbunches, the radiation frequency can be broadly tunable. According to our studies, the radiation frequency can be tuned from $0.1 \mathrm{THz}$ to above $1.0 \mathrm{THz}$, and the peak power of the radiation pulse can be up to the order of Watt, which is much higher than that of other $\mathrm{THz}$ sources based on the nonrelativistic electron beam.

\section{PRINCIPLE OF THE PROPOSED SCHEME}

Figure 1 shows the cross section of the proposed scheme. An electron bunch train is initiated on the photocathode of a DC gun by a laser pulse train with a time spacing of $\Delta \tau$, 
and subsequently accelerated by the adjustable high voltage (within the range of 90 to $200 \mathrm{kV}$, while the gun can work at tens to hundreds of $\mathrm{kV}[34,35]$ ) applied between the cathode and anode. A focusing electrode is used in the gun to confine the electron beam. Narrow-band $\mathrm{THz}$ radiation is excited as the electron bunch train traveling through the DLW. In the following, a brief introduction to the DLW wakefield radiation is presented.

DLW is a hollow cylindrical dielectric tube coated with metal on the outer surface. The dispersion equation describing the transverse modes of the DLW structure, for the azimuthally symmetric $\mathrm{TM}_{0 n}$ case, is given by [36]

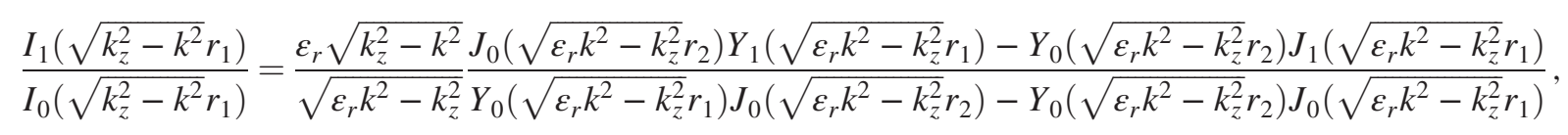

where $k=\frac{\omega}{c}$ and $k_{z}$ are the total wave number in the vacuum region and the longitudinal wave number respectively, $\varepsilon_{r}$ is the relative permittivity of the dielectric material, $J_{m}(x)$ and $Y_{m}(x)$ are Bessel functions of the first and second kinds of order $\mathrm{m}$, and $I_{m}(x)$ is the modified Bessel function of the first kind. The center frequencies and wave numbers $\left(\omega_{n}, k_{z n}\right)$ of the excited resonant modes $\mathrm{TM}_{0 n}$ are found from Eq. (1) by using the fact that they have phase velocity $\frac{\omega}{k_{z}}$ equal to the electron beam velocity $\beta c$. For a DLW with $r_{1}=0.4 \mathrm{~mm}, r_{2}=0.9 \mathrm{~mm}$ and $\varepsilon_{r}=$ 3.8 (material: fused silica), Fig. 2 shows the dispersion curves of the first four order modes and electron beam lines with different energies. One can find that the center frequencies can be tuned by adjusting the beam energy when the electron is nonrelativistic, but not for the accelerator-based relativistic electron beams, since their beam lines are almost the same as the light line drawn in Fig. 2.

The DLW is one kind of traveling wave tube (TWT) which can occur in various configurations: helix, diskloaded waveguide (coupled cavities), dielectric loaded waveguide, gratings, dielectrically coated metal and others. According to Pierce's theory [37], the coupling between the electromagnetic wave and the electron beam can be well described in terms of interaction impedance, which depends on the beam velocity, the geometry, and parameters of TWT structure. For the wakefield excited by the beam, the so-called loss factor is sometimes used

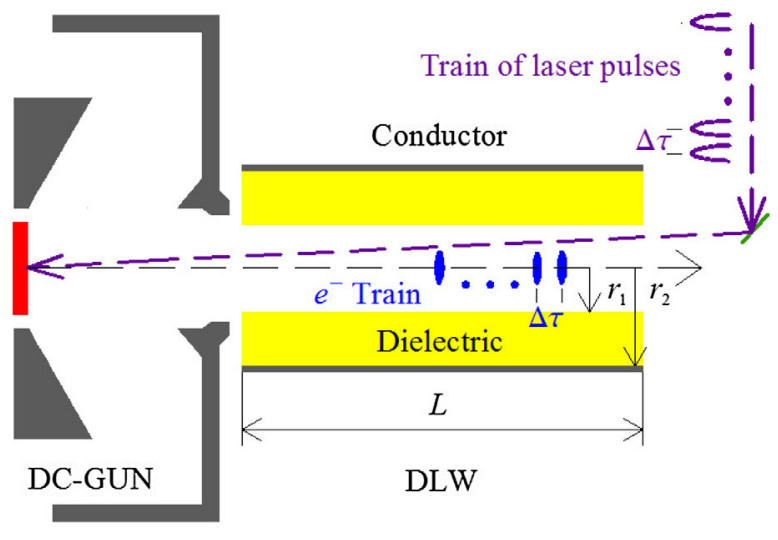

FIG. 1. Cross section draw of the proposed scheme. alternatively, which is closely related to Pierce's interaction impedance parameter and defined by [38]

$$
\kappa_{n}=\frac{\left|E_{n 0}\right|^{2}}{4 U_{n}\left(1-\frac{v_{g n}}{\beta c}\right)}
$$

with the unit of $\mathrm{V} /(\mathrm{mC})$, where $E_{n 0}$ is synchronous component of the longitudinal field on axis, $U_{n}$ is the stored energy per unit length in the mode, and the group velocity $v_{g n}$ is the derivative of the dispersion curve at the intersection point with the beam line. The explicit expression for the DLW can be obtained by following the methodology described in [39]

$$
\kappa_{n}=\frac{1}{4 \pi \varepsilon_{0}\left[\int_{0}^{r_{1}} r e_{z, n}^{2}(r) d r+\int_{r_{1}}^{r_{2}} r \varepsilon_{r} e_{z, n}^{2}(r) d r\right]},
$$

in which

$$
e_{z}(r)=\left\{\begin{array}{ll}
I_{0}\left(k_{n} r / \beta \gamma\right), & r<r_{1} \\
I_{0}\left(k_{n} r_{1} / \beta \gamma\right) \frac{F(r)}{F\left(r_{1}\right)}, & r_{1}<r<r_{2}
\end{array},\right.
$$

where

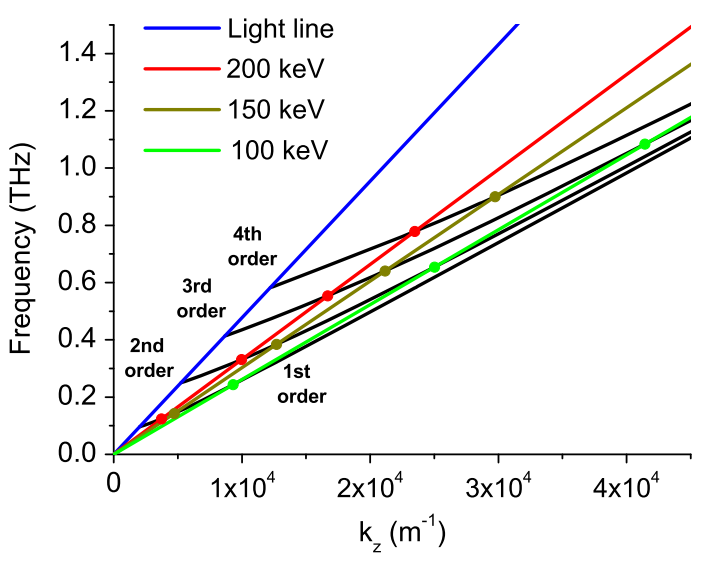

FIG. 2. Dispersion curves of the first four order modes (black lines) and electron beam lines with different energies. 


$$
F(r)=J_{0}\left(r k_{n}^{I I}\right) Y_{0}\left(r_{2} k_{n}^{I I}\right)-J_{0}\left(r_{2} k_{n}^{I I}\right) Y_{0}\left(r k_{n}^{I I}\right)
$$

with $k_{n}^{I I}=k_{n} \sqrt{\varepsilon_{r}-1 /-\beta^{2}}$.

The pulse power along the DLW structure is calculated by integrating the longitudinal component of the timeaveraged Poynting vector over the cross section of the structure. For a single electron case, the peak radiation power in time domain of the finite duration radiation pulse averaged over optical frequencies at a certain $\mathrm{TM}_{0 n}$ mode can be written as $[40,41]$

$$
\left(P_{0 n}\right)_{1}=\beta c \kappa_{n} e^{2} \frac{v_{g n}}{\beta c-v_{g n}} .
$$

This expression does not reflect the spectral contents of the radiation pulse, and a spectral analysis would result in the radiation bandwidth. For a single electron bunch with length shorter than the radiation wavelength, the radiation can be coherently enhanced, which depends on the coherence factor of electron bunch. When considering an electron bunch train as driving source, the spectral power would involve a product with the bunch train spectral coherence factor.

For an electron beam consisting of $N_{b}$ repetitive Gaussian microbunches with temporal spacing $\Delta \tau$, the coherence factor can be written as [25]

$$
\alpha(\omega)=\frac{1}{N_{b}}\left|\frac{\sin \frac{N_{b} \Delta \tau}{2} \omega}{\sin \frac{\Delta \tau}{2} \omega}\right| \exp \left(-\frac{\omega^{2} \sigma_{\tau}^{2}}{2}\right)
$$

where $\sigma_{\tau}$ is the rms length of the microbunch. The coherence factor is resonant at $\omega_{m}=2 m \pi / \Delta \tau(m=1,2, \ldots)$ with a bandwidth:

$$
\frac{\Delta \omega_{m}}{\omega_{m}}=\frac{1}{m N_{b}} .
$$

Wakefields at one mode will be enhanced if its center frequency $\omega_{n}$ belongs to the coherence spectrum, namely $\omega_{n}$ happens to be near one of the resonant frequencies $\omega_{m}$.

To make the excited wakefield enhanced by all the electrons, the tail of beam should reach the wakefield region generated by the head of beam in the DLW structure, thus the DLW length $L$ must hold the condition:

$$
L>\frac{L_{B}}{\left(\beta c / v_{g n}-1\right)},
$$

where $L_{B}$ is the total beam length.

From the above, the tunable $\mathrm{THz}$ radiation source can be obtained by properly choosing the beam energy and the time spacing $\Delta \tau$ of the electron bunch train.

\section{GENERATION OF ELECTRON BUNCH TRAIN}

In this section, we would like to investigate the production of an electron bunch train. To generate a train of electron bunches with tunable time spacing, the key is to obtain a train of laser pulses with tunable repetition rate, which has been conceptually proposed and experimentally studied in the previous works $[22,23,42,43]$. An interferometer-based pulse stacking method is preferred in this scheme, and the principle of this method can be found in Fig. 4 of Ref. [23]. After $n$ stages stacking, $2^{n}$ micropulses
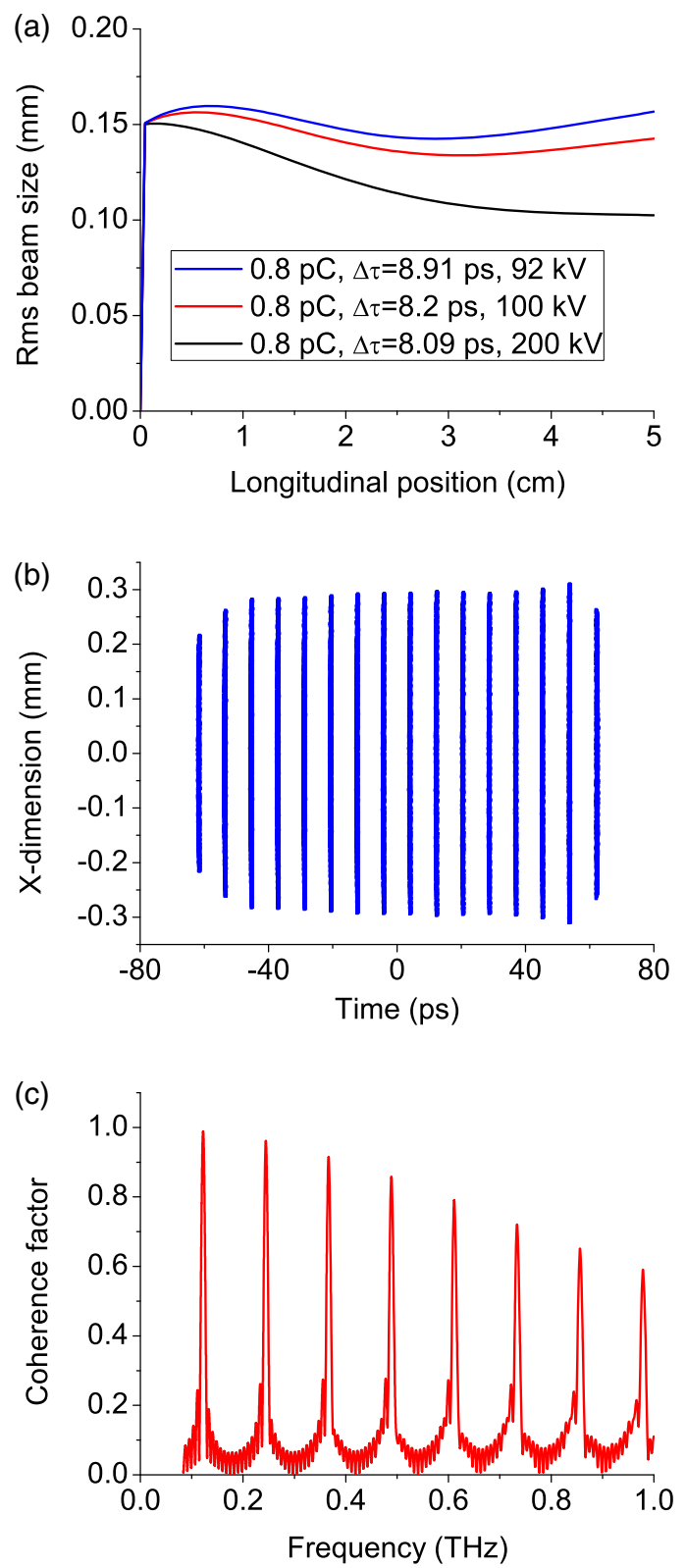

FIG. 3. Electron beam simulation results: (a) the evolutions of rms beam size along the longitudinal position; (b)the cross section distribution state and (c) the coherence factor of the electron beam at the DLW entrance position when the initial time spacing $\Delta \tau=8.2 \mathrm{ps}$ and gun voltage is $100 \mathrm{kV}$. 
can be generated, and the time spacing $\Delta \tau$ can be adjusted continuously by the optical delay lines. Meanwhile the beam energy can be easily adjusted by changing the gun voltage.

For an initial electron beam with 16 microbunches, $0.8 \mathrm{pC}$ charge $(50 \mathrm{fC} \times 16), 0.3 \mathrm{~mm}$ radius and $100 \mathrm{fs}$ rms microbunch length, 3D beam dynamics including space charge force is simulated by using code ASTRA [44], and the results are shown in Fig. 3. For experimental practice, a photocathode with high quantum efficiency (QE) should be adopted to mitigate the power requirement of the driving laser. The magnesium cathode could be appropriate for its relatively high QE and long lifetime [45]. In the simulation, a DC gun with a $4.5 \mathrm{~cm}$ accelerating gap and a $0.5 \mathrm{~cm}$ drift are considered. The electron beam is focused by the focusing electrode of the gun, and the focal length is dependent on the gun voltage. Figure 3(a) shows the evolutions of the rms beam size along the longitudinal position. At the entrance of the DLW (at the position of $5 \mathrm{~cm}$ ), the beam radius is smaller than the inner radius of the DLW. The cross section distribution state and coherence factors of the $100 \mathrm{keV}$ electron beam at the DLW entrance are shown in Fig. 3(b) and (c), and the coherence factors are still very high at harmonics, which means that a small variation in $\Delta \tau$ gives rise to a broad tuning range for the radiation frequency.

In addition to the space charge force and the transverse emittance of the electron beam, the wakefield excited in the DLW can also induce beam divergence, which might cause particle loss in the DLW structure. In the following section, the beam transport will be discussed.

\section{BEAM TRANSPORT IN THE DLW}

Electron loss in the dielectric will result in asymmetric charging of the dielectric, leading to beam deflection by the static electric field generated, correspondingly more charging and finally the structure breakdown [46]. Technology

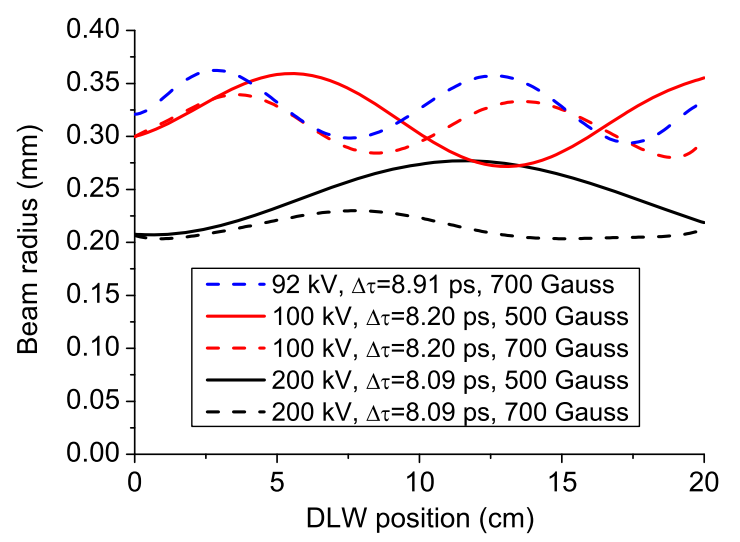

FIG. 4. Beam radius evolutions along the longitudinal DLW position. for charging prevention is considered for high energy wakefield accelerators $[47,48]$.

For studying the electron beam transport through the DLW structure and the wakefield radiation excited by the electron bunch trains as well, the start-to-end simulation is carried out. The ASTRA simulated macroparticles are imported into 3D electromagnetic particle-in-cell code CST [49] at the entrance of the DLW. A $20 \mathrm{~cm}$ long DLW structure is modeled, and an external solenoid field is used to confine and guide the electron beam. The inner and outer radius, and the relative permittivity of the DLW are $0.4 \mathrm{~mm}, 0.9 \mathrm{~mm}$, and 3.8 respectively, and the charge of driving electron beam is $50 \mathrm{fC} \times 16=0.8 \mathrm{pC}$ as presented in the previous section. According to the simulation results shown in Fig. 4: for the electron beam with energy greater than $100 \mathrm{keV}$, the radius is smaller than the inner radius of the DLW structure along the whole longitudinal position when a 500 Gauss solenoid field is applied; and a
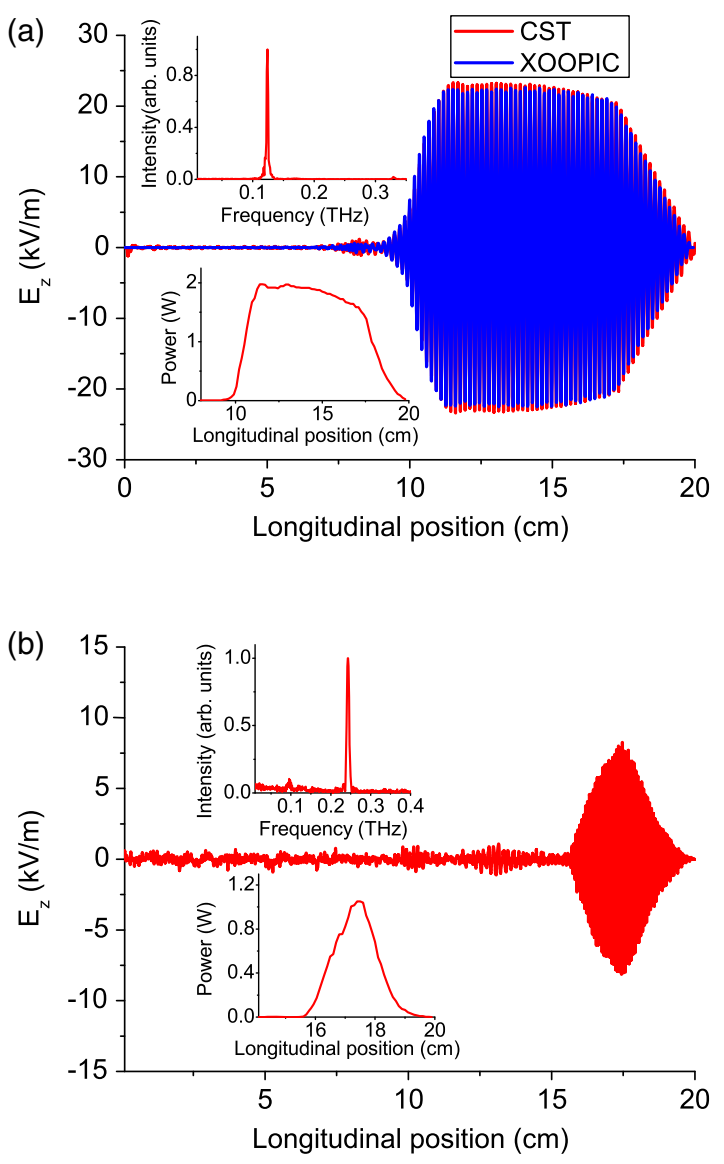

FIG. 5. Simulated electric field strength at $r=0.40 \mathrm{~mm}$, the corresponding spectrum calculated by Fourier transform, and the pulse power distribution along the longitudinal position. (a) The coherence factor is resonant at $\mathrm{TM}_{01}$ mode $\left(\Delta \tau=\frac{1}{0.1236 \mathrm{THz}}=8.09 \mathrm{ps}\right)$ and the beam energy is $200 \mathrm{keV}$; (b) The coherence factor is resonant at $\mathrm{TM}_{01}$ mode $\left(\Delta \tau=\frac{1}{0.2434 \mathrm{THz}} \cdot 2=8.2 \mathrm{ps}\right)$ and the beam energy is $100 \mathrm{keV}$. 


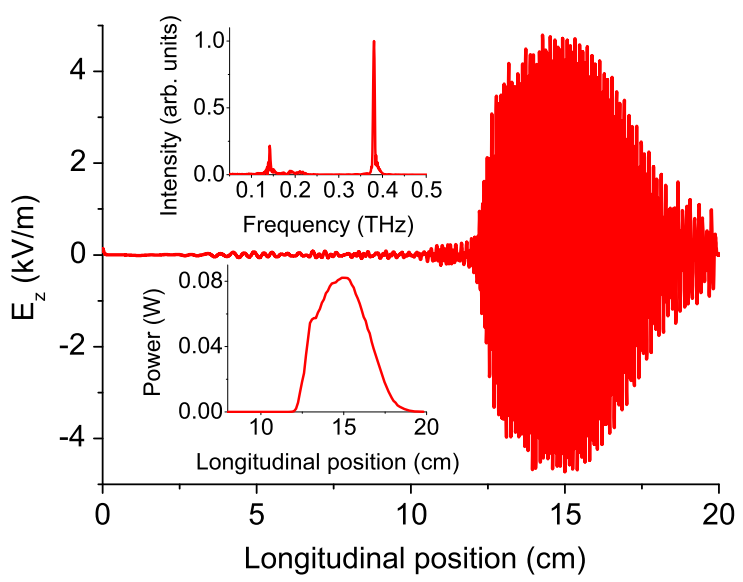

FIG. 6. The same as that of Fig. 5, when the coherence factor is resonant at $\mathrm{TM}_{02}$ mode $\left(\Delta \tau=\frac{1}{0.3842 \mathrm{THz}} \cdot 2=5.2 \mathrm{ps}\right)$ and the beam energy is $150 \mathrm{keV}$.

700 Gauss field should be required for the transport of electron beam at $92 \mathrm{keV}$.

\section{SIMULATION RESULTS OF THE RADIATION}

The simulation results of wakefield radiation excited by electron bunch trains used in the above section are presented in Fig. 5, where the solenoid filed is 500 Gauss. The simulated radiation frequencies are consistent with the theory predictions. In Fig. 5, the longitudinal electric field simulated by the 2D particle-in-cell code XOOPIC [50] is also presented, which agree well with the simulation result of the code CST.

For the radiation of high order mode, simulation results of $\mathrm{TM}_{02}$ mode for electron bunch train at $150 \mathrm{keV}$ are shown in Fig. 6 as an example. Since the loss factor $\kappa_{n=2}$ is much smaller than $\kappa_{n=1}$, the first order mode radiation still exists. The simulation results for electron bunch trains with different energies and time spacings are summarized in Table I.

TABLE I. Radiation parameters for different bunch trains.

\begin{tabular}{lccc}
\hline \hline Modes & e-bunch $(\mathrm{keV}, \mathrm{ps})$ & $f(\mathrm{THz})$ & $\mathrm{P}^{\mathrm{a}}(\mathrm{mW})$ \\
\hline $\mathrm{TM}_{01}$ & $200, \Delta \tau=8.09$ & 0.1236 & $2.06 \times 10^{3}$ \\
& $150, \Delta \tau=7.0$ & 0.1427 & $1.98 \times 10^{3}$ \\
& $100, \Delta \tau=4.10 \times 2$ & 0.2434 & $1.05 \times 10^{3}$ \\
& $92, \Delta \tau=2.97 \times 3$ & 0.3369 & $1.53 \times 10^{2}$ \\
$\mathrm{TM}_{02}$ & $200, \Delta \tau=3.02 \times 2$ & 0.3309 & $2.73 \times 10^{2}$ \\
& $150, \Delta \tau=2.6 \times 2$ & 0.3842 & 82.7 \\
$\mathrm{TM}_{03}$ & $100, \Delta \tau=1.53 \times 4$ & 0.6535 & 4.99 \\
& $200, \Delta \tau=1.81 \times 3$ & 0.5543 & 18.52 \\
& $150, \Delta \tau=1.57 \times 4$ & 0.6407 & 4.2 \\
\hline \hline
\end{tabular}

apeak pulse power at certain frequency.

\section{SUMMARY AND DISCUSSION}

In this paper, we propose a novel scheme to generate tunable narrow-band $\mathrm{THz}$ radiation from a DLW structure excited by nonrelativistic electron bunches. The radiation frequency can be tuned from $0.1 \mathrm{THz}$ to above $1 \mathrm{THz}$, and the power is relatively high.

The radiation structure used in this scheme can be a pulled fused silica capillary tube, which is easily fabricated; the driving laser of the DC gun is commercially available and can work at $100 \mathrm{MHz}$ repetition rate, which means that a high average power radiation source can be obtained; the DC gun is also a mature technology. So the proposed scheme is very robust and can easily be achieved. Although there is more compact and commercially available source, such as the GaAs Schottky diode, which can offer a $1 \mathrm{~mW}$ continuous wave at $1 \mathrm{THz}$, one might note that the proposed source is more broadly tunable in frequency and has a calculated output power that is a factor of ten higher. To extract the radiation, circular waveguide elements immediately following the DLW tube can be used to control the diverging $\mathrm{THz}$ beam and ultimately direct the radiation forward from a horn antenna toward the mirrors [16].

In addition, the power can be enhanced by increasing the number of microbunches $N_{b}$. However, the bunch train jitter from the laser stacking process to forming a micropulse train may cause a misalignment between the coherence spectrum and the radiation spectrum of DLW modes. Therefore the number of micropulses should be chosen appropriately according to the precision of optical elements considering the fact that the bandwidth of the coherence spectrum is $1 / m N_{b}$.

\section{ACKNOWLEDGMENTS}

This work is supported by Fundamental Research Funds for the Central Universities under Contracts No. WK2310000042 and No. WK2310000047, Natural Science Foundation of China under Contracts No. 11205152, No. 11375199, and No. 11175182.

[1] G. P. Williams, Filling the THz gap-high power sources and applications, Rep. Prog. Phys. 69, 301 (2006).

[2] M. Tonouchi, Cutting-edge terahertz technology, Nat. Photonics 1, 97 (2007).

[3] Q. Qin, B. S. Williams, S. Kumar, J. L. Reno, and Q. Hu, Tuning a terahertz wire laser, Nat. Photonics 3, 732 (2009).

[4] G. L. Carr, M. C. Martin, W. R. McKinney, K. Jordan, G. R. Neil, and G. P. Williams, High-power terahertz radiation from relativistic electrons, Nature (London) 420, 153 (2002).

[5] M. Abo-Bakr, J. Feikes, K. Holldack, P. Kuske, W. B. Peatman, U. Schade, G. Wustefeld, and H. W. Hubers, Brilliant, Coherent Far-Infrared (THz) Synchrotron Radiation, Phys. Rev. Lett. 90, 094801 (2003). 
[6] J. Barros, C. Evain, L. Manceron et al., Coherent synchrotron radiation for broadband terahertz spectroscopy, Rev. Sci. Instrum. 84, 033102 (2013).

[7] W. P. Leemans et al., Observation of Terahertz Emission from a Laser-Plasma Accelerated Electron Bunch Crossing a Plasma-Vacuum Boundary, Phys. Rev. Lett. 91, 074802 (2003).

[8] A. Perucchi, S. Di Mitri, G. Penco, E. Allaria, and S. Lupi, The TeraFERMI terahertz source at the seeded FERMI free-electron-laser facility, Rev. Sci. Instrum. 84, 022702 (2013).

[9] Z. Wu, A.S. Fisher, J. Goodfellow, M. Fuchs, D. Daranciang, M. Hogan, H. Loos, and A. Lindenberg, Intense terahertz pulses from SLAC electron beams using coherent transition radiation, Rev. Sci. Instrum. 84, 022701 (2013).

[10] D. Wang, L. X. Yan, Y. C. Du, J. F. Hua, Q. Du, H. J. Qian, X. H. Lu, W. H. Huang, H. B. Chen, and C.X. Tang, Generating ultrabroadband terahertz radiation based on the under-compression mode of velocity bunching, Rev. Sci. Instrum. 84, 022704 (2013).

[11] R. Prazeres, F. Glotin, J. M. Ortega, C. Rippon, R. Andouart, J. M. Berset, E. Arnaud, and R. Chaput, Study of the "CLIO" FEL properties at long wavelengths, Nucl. Instrum. Methods Phys. Res., Sect. A 445, 204 (2000).

[12] B. L. Militsyn and A. F. G. van der Meer, Design Aspects Of Felice, The Free Electron Laser For Intra-Cavity Experiments, in Proceedings of the 8th European Particle Accelerator Conference, Paris, 2002 (EPS-IGA and CERN, Geneva, 2002), p. 819.

[13] W. Schöllkopf, S. Gewinner, W. Erlebach et al., The new IR Fel Facility at The Fritz-Haber-Institut in Berlin, Proceedings of FEL2014, Basel, Switzerland, WEBO4 (JACoW, Basel, 2014).

[14] M. Yokoyama, F. Oda, K. Nomaru, H. Koike, M. Sobajima, M. Kawai, H. Kuroda, and K. Nakai, Improvement of KHI FEL device at FEL-SUT, Nucl. Instrum. Methods Phys. Res., Sect. A 507, 261 (2003).

[15] Y. C. Huang, Desktop megawatt superradiant free-electron laser at terahertz frequencies, Appl. Phys. Lett. 96, 231503 (2010).

[16] A. M. Cook, R. Tikhoplav, S. Y. Tochitsky, G. Travish, O. B. Williams, and J.B. Rosenzweig, Observation of Narrow-Band Terahertz Coherent Cherenkov Radiation from a Cylindrical Dielectric-Lined Waveguide, Phys. Rev. Lett. 103, 095003 (2009).

[17] S. Antipov, C. Jing, P. Schoessow, A. Kanareykin, V. Yakimenko, A. Zholents, and W. Gai, High power terahertz radiation source based on electron beam wakefields, Rev. Sci. Instrum. 84, 022706 (2013).

[18] A. V. Smirnov, R. Agustsson, W. J. Berg et al., Observation of a variable sub- $\mathrm{THz}$ radiation driven by a low energy electron beam from a thermionic rf electron gun, Phys. Rev. ST Accel. Beams 18, 090703 (2015).

[19] G. Stupakov, Using pipe with corrugated walls for a subterahertz free electron laser, Phys. Rev. ST Accel. Beams 18, 030709 (2015).

[20] S. E. Korbly, A. S. Kesar, J. R. Sirigiri, and R. J. Temkin, Observation of Frequency-Locked Coherent Terahertz
Smith-Purcell Radiation, Phys. Rev. Lett. 94, 054803 (2005).

[21] A. S. Kesar, R. A. Marsh, and R. J. Temkin, Power measurement of frequency-locked Smith-Purcell radiation, Phys. Rev. ST Accel. Beams 9, 022801 (2006).

[22] Y. Li, Y. E. Sun, and K. J. Kim, High-power beam-based coherently enhanced $\mathrm{THz}$ radiation source, Phys. Rev. ST Accel. Beams 11, 080701 (2008).

[23] Z. He, Y. Xu, W. Li, and Q. Jia, Generation of quasiequally spaced ultrashort microbunches in a photocathode if gun, Nucl. Instrum. Methods Phys. Res., Sect. A 775, 77 (2015).

[24] B. Marchetti, A. Bacci, E. Chiadroni, A. Cianchi, M. Ferrario, A. Mostacci, R. Pompili, C. Ronsivalle, B. Spataro, and I. Zagorodnov, Novel schemes for the optimization of the SPARC narrow band THz source, Rev. Sci. Instrum. 86, 073301 (2015).

[25] A. Gover, Superradiant and stimulated-superradiant emission in prebunched electron-beam radiators. I. Formulation, Phys. Rev. ST Accel. Beams 8, 030701 (2005).

[26] I. Schnitzer and A. Gover, The prebunched free electron laser in various operating gain regimes, Nucl. Instrum. Methods Phys. Res., Sect. A 237, 124 (1985).

[27] Y. Li and K.-J. Kim, Nonrelativistic electron bunch train for coherently enhanced terahertz radiation sources, Appl. Phys. Lett. 92, 014101 (2008).

[28] M. Mineo and C. Paoloni, Corrugated Rectangular Waveguide Tunable Backward Wave Oscillator for Terahertz Applications, IEEE Trans. Electron Devices 57, 1481 (2010).

[29] W. Gai, P. Schoessow, B. Cole, R. Konecny, J. Norem, J. Rosenzweig, and J. Simpson, Experimental Demonstration of Wake-Field Effects in Dielectric Structures, Phys. Rev. Lett. 61, 2756 (1988).

[30] J. G. Power, M. E. Conde, W. Gai, R. Konecny, P. Schoessow, and A. D. Kanareykin, Measurements of the longitudinal wakefields in a multimode, dielectric wakefield accelerator driven by a train of electron bunches, Phys. Rev. ST Accel. Beams 3, 101302 (2000).

[31] A. Zholents, W. Gai, S. Doran et al., A preliminary design of the collinear dielectric wakefield accelerator, Nucl. Instrum. Methods Phys. Res., Sect. A 829, 190 (2016).

[32] S. Antipov, C. Jing, M. Fedurin, W. Gai, A. Kanareykin, K. Kusche, P. Schoessow, V. Yakimenko, and A. Zholents, Experimental Observation of Energy Modulation in Electron Beams Passing through Terahertz Dielectric Wakefield Structures, Phys. Rev. Lett. 108, 144801 (2012).

[33] W. Li, Z. He, and Q. Jia, Electron bunch length measurement with a wakefield radiation decelerator, Phys. Rev. ST Accel. Beams 17, 042801 (2014).

[34] J. Urata, M. Goldstein, M. F. Kimmitt, A. Naumov, C. Platt, and J. E. Walsh, Superradiant Smith-Purcell Emission, Phys. Rev. Lett. 80, 516 (1998).

[35] K. Togawa, T. Shintake, T. Inagaki, K. Onoe, T. Tanaka, H. Baba, and H. Matsumoto, $\mathrm{CeB}_{6}$ electron gun for lowemittance injector, Phys. Rev. ST Accel. Beams 10, 020703 (2007).

[36] T.-B. Zhang, T. Marshall, and J. Hirshfield, A Cerenkov source of high-power picosecond pulsed microwaves, IEEE Trans. Plasma Sci. 26, 787 (1998). 
[37] J. R. Pierce, Traveling-Wave Tubes (D. van Nostrand Company Inc., Princeton, NJ, 1950).

[38] K. L. F. Bane and G. Stupakov, Impedance of a rectangular beam tube with small corrugations, Phys. Rev. ST Accel. Beams 6, 024401 (2003).

[39] S. Y. Park and J. L. Hirshfield, Theory of wakefields in a dielectric-lined waveguide, Phys. Rev. E 62, 1266 (2000).

[40] F. Gao, M. E. Conde, W. Gai, C. Jing, R. Konecny, W. Liu, J. G. Power, T. Wong, and Z. Yusof, Design and testing of a $7.8 \mathrm{GHz}$ power extractor using a cylindrical dielectricloaded waveguide, Phys. Rev. ST Accel. Beams 11, 041301 (2008).

[41] D. Wang et al., High Power Rf Radiation At W-Band Based On Wakefields Excited By Intense Electron Beam, in Proceedings of the 6th International Particle Accelerator Conference, Richmond, VA, USA (JACoW, Richmond, 2015), p. 2960.

[42] C. W. Siders, J. L. W. Siders, A. J. Taylor, S.-G. Park, and A. M. Weiner, Efficient high-energy pulse-train generation using a $2^{n}$-pulse michelson interferometer, Appl. Opt. 37, 5302 (1998).

[43] Y. Shen, X. Yang, G. L. Carr, Y. Hidaka, J. B. Murphy, and X. Wang, Tunable Few-Cycle and Multicycle Coherent
Terahertz Radiation from Relativistic Electrons, Phys. Rev. Lett. 107, 204801 (2011).

[44] K. Flottman, ASTRA user manual, http://www.desy.de/ $\sim$ mpyflo/.

[45] T. Rao and D.H. Dowell, An Engineering Guide To Photoinjectors, arXiv:1403.7539.

[46] C. Jing, J. Power, and A. Zholents, Dielectric Wakefield Accelerator to drive the future FEL Light Source, Report No. ANL/APS/LS-326, 2011.

[47] S. V. Shchelkunov, T. C. Marshall, and J. L. Hirshfield, Diagnostic and Detectors for Charging and Damage of Dielectrics in High-gradient Accelerators, arXiv: $1510.05507 \mathrm{v} 1$.

[48] P. Schoessow, S. P. Antipov, C. Jing, A. Kanareykin, S. Zuo, J. G. Power, and A. Zholents, Analysis of High Repetition Rate Effects in Dielectric Wakefield Accelerators, in Proceedings of the 25th Particle Accelerator Conference, PAC-2013, Pasadena, CA, 2013 (IEEE, New York, 2013), p. 93.

[49] CST Corp., CST PS Tutorials, http://www.cst-china.cn/.

[50] J. P. Verboncoeur, A. B. Langdon, and N. T. Gladd, An object-oriented electromagnetic PIC code, Comput. Phys. Commun. 87, 199 (1995). 